\title{
Covid-19: La brecha (digital) educativa a través de la prensa
}

\section{Covid-19: Educational (digital) divide through the press}

\author{
Belén Suárez Lantarón \\ Universidad de Extremadura (España) \\ bslantaron@unex.es \\ Nuria García-Perales \\ Universidad de Extremadura (España) \\ nuria@unex.es
}

Recibido: 06/04/2021

Aceptado: 20/09/2021

Publicado: 01/12/2021

\section{RESUMEN}

La situación socio-sanitaria ocasionada por la COVID-19 ha implicado la clausura de los centros educativos forzando a la población escolar a continuar su proceso de enseñanza-aprendizaje de forma virtual. Esta situación ha puesto en evidencia la brecha digital existente entre los escolares, una realidad que ha aflorado en toda su magnitud para convertirse en objeto y tema de debate en el ámbito periodístico. Este trabajo tiene por objetivo describir el alcance de la brecha digital en el ámbito educativo y sus consecuencias, así como las posibles soluciones a corto y largo plazo. La metodología elegida es de carácter cualitativo apoyada en el análisis de contenido, en la que se exploran y revisan publicaciones de prensa generalista española en el primer mes de confinamiento, tanto de ámbito nacional como autonómico. Los resultados muestran que la prensa aborda el desequilibrio y desigualdad social respecto al acceso a la información y las dificultades consecuentes para acceder a la modalidad online, se observa un claro interés por la brecha digital, principalmente de acceso a internet, como una más de las dificultades en entornos socio-económicos vulnerables para acceder a la educación en igualdad de condiciones. Como conclusión cabe destacar que la pandemia, a través de la prensa generalista, ha incrementado la visibilidad de una situación ya existente: la desigualdad digital. Una desigualdad que favorece la exclusión en una sociedad que se muestra eminentemente tecnológica.

\section{PALABRAS CLAVE}

Educación; brecha digital; prensa; COVID-19.

\section{ABSTRACT}

The socio-sanitary situation caused by COVID-19 has implied the closure of educational centers, forcing the school population to continue their teaching-learning process virtually. This situation has highlighted the existing digital divide between schoolchildren, a reality that has emerged in all its magnitude to become an object and topic of debate in the journalistic field. This work aims to describe the scope of the digital divide in education and its consequences, as well as possible solutions in the short and long term. The chosen methodology is of a qualitative nature supported by content analysis, in which general Spanish press publications are explored and reviewed in the first month of confinement, both nationally and regionally. The results show that the press addresses the social imbalance and inequality regarding access to information and the consequent difficulties in accessing the online modality, there is a clear interest in the digital divide, mainly internet access, as one more of the Difficulties in vulnerable socio-economic environments to access education under equal conditions. In conclusion, it should be noted that the pandemic, through the generalist press, has increased the visibility of an already existing situation: digital inequality, which favors exclusion in a society that is eminently technological.

\section{KEYWORDS}

Education; digital divide; press; COVID-19. 


\section{CITA RECOMENDADA}

Suárez, B. \& García-Perales, N. (2021). Covid-19: La brecha (digital) educativa a través de la prensa. RIITE Revista Interuniversitaria de Investigación en Tecnología Educativa, 11, 53-68. https://doi.org/10.6018/riite.475881

\section{Principales aportaciones del artículo y futuras líneas de investigación:}

- Este trabajo aporta reconocimiento sobre un hecho social: la brecha digital; considerando esta una cuestión relevante como así lo demuestra la preocupación manifestada por los medios de comunicación en el primer mes de confinamiento. Esta brecha también se sostiene en el ámbito educativo donde puede provocar diferencias o desigualdades que afectan al presente, pero también al futuro del alumnado, en muchas ocasiones, más vulnerable. Analizar sus causas y consecuencias puede ayudar a comprender dicho fenómeno.

- Como cierre, se destaca la función de la escuela como institución frente a las desigualdades y se proponen algunas acciones o posibles soluciones para la construcción de una realidad digital inclusiva. Por ello, las futuras líneas de investigación que se proponen son: a) realizar estudio para comparar, confrontar y cotejar estos resultados con datos obtenidos a través de estudios publicados en revistas científicas; b) extender la obtención de datos en temporalidad, analizando documentos que abarquen todo el periodo en el que se ha mantenido la educación online y c) comparar con países del entorno cómo se ha abordado por parte de la prensa esta situación.

\section{INTRODUCCIÓN}

La covid-19 provocó la clausura de las instituciones educativas, tanto aquellas que integran la red del sistema educativo formal como aquellas otras que desarrollan su labor desde el ámbito no-formal, y forzó al alumnado a seguir su formación en un entorno virtual. Sin embargo, ante las numerosas posibilidades que ofrece lo digital, como la democratización del acceso a la información y el conocimiento, la eclosión de ecologías de aprendizaje ubicuo y el vertiginoso desarrollo de programas, plataformas y aplicaciones susceptibles de ser utilizadas con éxito en el ámbito educativo (Díez y Díaz, 2018; Burbules, 2014), surge el inquietante abismo de la brecha digital, que se constituye como una nueva desigualdad estructural que incide sobre la pobreza y la exclusión y que, asimismo, es reflejo de desigualdades sociales previas (Olarte, 2017; Alva, 2015).

Autores como Cabero y Palacios (2019) señalan que el concepto de brecha digital comprende dos dimensiones genéricas: por un lado, la imposibilidad de acceder a la información que ofrece Internet y, por otro, la dificultad de alcanzar la competencia digital, es decir, de disponer de las habilidades suficientes y necesarias para informarse, comunicarse, crear contenidos, navegar con seguridad y resolver problemas.

Fruto de la evolución e incursión de la tecnología en casi todos los ámbitos de la sociedad, el concepto se ha redefinido al objeto de explicar sus orígenes, propiedades, efectos e impacto social (Burri, 2011; Cabero y Ruiz, 2018 o Fernández, 2017) excediendo su componente tecnológico para instalarse en la "apropiación social" (Andrés, 2014, p.18) bajo el análisis de las dimensiones socio-económica, praxiológica, subjetivo-individual, socio-cultural y política (Gómez-Navarro et al. 2018). Así, la brecha digital no se define como un todo, sino como una realidad poliédrica compuesta de distintas dimensiones que pueden operar de manera individual o en interconexión.

Una dimensión es la brecha digital de acceso, la carencia de equipamiento y/o de conexión a Internet. La calidad y tipo de los dispositivos determina tanto las condiciones de conexión como las tareas que pueden realizarse, por lo que la experiencia, en este caso educativa, puede verse condicionada por los dispositivos disponibles en el círculo familiar (Calderón, 2019; Rivoir y Escuder, 2018). Aspectos socio-económicos y geográficos pueden dificultar la conexión, siendo los sectores de población más desfavorecida y las zonas rurales 
y/o con mayores índices de despoblación los que cuentan con menor disponibilidad a un acceso de calidad (Cabero y Ruiz, 2018; Torres, 2017 o Vázquez, 2017). Se incluyen otros factores como pertenecer a comunidades de lenguas minoritarias, población migrante o la brecha digital de género (Keely, 2017; Tejada y Domínguez, 2018).

Otra dimensión es la brecha de uso: habilidades, competencias, actitudes y experiencias de empleo de las tecnologías. Disponer de equipos y conexión adecuados no implica franquear la brecha, puesto que es necesario haber alcanzado la competencia digital, entendida como la capacidad de hacer un uso seguro y crítico de las tecnologías de la información, comunicación y resolución de problemas básicos en todos los aspectos de la vida (Carneiro, Toscano y Díaz, 2011).

Por último, debe tenerse en cuenta la brecha digital referida al aprovechamiento y calidad de uso de la tecnología, definida a través de las finalidades pragmáticas y los beneficios que esta reporta a la ciudadanía. De este modo, es preciso que la tecnología incida positivamente en la vida, desde lo social a lo académico, pasando por el ocio, la cultura o las relaciones con las instituciones. Una alfabetización digital que pasa por ser "mediática, digital, multimodal, crítica y funcional" (Gutiérrez y Tyner, 2011, p. 31).

Ambas cuestiones, la existencia de una brecha digital y la obligatoriedad de realizar la formación de forma virtual que puede ocasionar desventajas o exclusión entre los escolares, se ven reflejadas como actualidad mediática, como muestra el barómetro del Centro de Investigaciones sociológicas (CIS) realizado durante el mes de marzo, en el que se observa un repunte en cuanto a considerar la educación como uno de los principales problemas en España (datos que se pueden consultar en: http://www.cis.es/).

\section{OBJETIVO}

La situación antes descrita ha conseguido exponer de forma más evidente la brecha digital ya existente, la cual ha sido y es foco del interés científico desde el ámbito educativo. Sin embargo, se observa un vacío respecto a estudios en los que se utilice como fuente la prensa y su perspectiva mediática, máxime considerando el papel que estos medios desempeñan en la construcción de la realidad social y su consecuente influencia en las agendas públicas y/o políticas.

Estas cuestiones motivan el desarrollo de este trabajo, cuyo objetivo es revisar y describir el interés generado sobre la brecha digital en la prensa generalista desde una perspectiva socio-educativa. De la observación, análisis e interpretación de los datos se pretende construir el relato que, sobre la brecha digital en el ámbito educativo español, se traslada desde los medios de comunicación a la población.

\section{MÉTODO}

Entendida la brecha digital como un fenómeno social en cuya naturaleza confluyen aspectos económicos, políticos, culturales o educativos, se aborda este estudio desde una perspectiva cualitativa apoyada en el análisis de contenido, método adecuado para generar conocimiento sobre un hecho social puesto que se trata de un método sensible a las diferencias, los casos particulares, el cambio social y el educativo (Albert, 2009; Pérez, 2011).

Nos situamos en un papel de observadores no participantes con un acceso indirecto al hecho estudiado -la brecha digital- al no mantener contacto directo con los sujetos y los fenómenos, sino que su conocimiento viene mediado y tamizado por la prensa.

Como fuente de obtención de información se elige la prensa escrita (en versión digital) considerando que transmiten a la ciudadanía, en forma de noticias, una selección de fragmentos de los acontecimientos que acaban por convertirse en la realidad social aceptada. Asimismo, justifica esta elección la Agenda Setting que señala el doble papel que tiene la prensa a la hora de seleccionar y publicar noticias: por un lado, tratando los temas que consideran más relevantes en cada momento o situación; por otro, marcando la opinión pública. 
La prensa es una ventana que deja ver lo que ocurre en contextos próximos y remotos a nuestra ubicación; sin embargo, el conocimiento que se obtiene es indirecto y mediado por el qué, cuándo y cómo deciden publicar (Califano, 2015). Consecuentemente, han de considerarse como una fuente de carácter secundario, ya que puede no ofrecer una realidad aséptica de los fenómenos sociales ocurridos, sino el resultado de la percepción e interpretación que de ellos hace el periodista o la línea editorial del medio.

En la selección de textos periodísticos se ha considerado que estos se presenten en formato digital y de libre acceso. El sistema de búsqueda ha sido manual, revisando diferentes medios de comunicación nacional y autonómicos Las palabras clave utilizadas para acotar la temática son: brecha digital y educación, las cuales deben aparecer en el titular de la noticia o en el subtítulo y/o cuerpo del texto. Además, se han considerado los siguientes criterios: (a) Temporal: artículos publicados durante el primer mes del estado de alarma en España (14 de marzo hasta 14 de abril de 2020), momento inicial del obligado traslado de la enseñanza al espacio virtual; (b) Tipo: prensa española de carácter generalista, tanto nacional como autonómica-local (ver tabla 1). Se rechazan aquellas publicaciones que, aun conteniendo las palabras clave, no atañen estrictamente a cuestiones educativas.

La muestra total la conforman 374 artículos, de los cuales 140 pertenecen a prensa de tirada nacional y 234 al ámbito autonómico, provincial o local. Las publicaciones se recogen en un escritorio online, creado con la herramienta Symbaloo. Se elige esta aplicación dado que proporciona el soporte para su registro, organización y fácil acceso o recuperación.

\section{Tabla 1. Grupos de comunicación y periódicos}

\begin{tabular}{|c|c|}
\hline Grupo de publicación & Periódicos \\
\hline Grupo Prisa & El País \\
\hline Grupo Vocento & $\begin{array}{l}\text { ABC; El Correo; El Diario Vasco; El Diario Montañés; El } \\
\text { Norte de Castilla; La voz de Cádiz; La Rioja; El Comercio; } \\
\text { Las Provincias; Hoy. }\end{array}$ \\
\hline Grupo Godó & La Vanguardia \\
\hline Prensa Ibérica (antes Grupo Zeta) & $\begin{array}{l}\text { La Nueva España; La Provincia Diario de Las Palmas; } \\
\text { Información; El Faro de Vigo; Diario de Córdoba; El } \\
\text { Periódico de Cataluña; El Periódico de Aragón; El } \\
\text { Periódico de Extremadura; El Periódico Mediterráneo; El } \\
\text { Día de Santa Cruz de Tenerife. }\end{array}$ \\
\hline Unidad Editorial & El Mundo \\
\hline Grupo Planeta & La Razón \\
\hline Editorial Ecoprensa & El Economista \\
\hline Display Connector & Público \\
\hline Diario de Prensa Digital S.L. & elDiario.es \\
\hline Grupo Henneo & $\begin{array}{l}\text { Heraldo de Aragón; } 20 \text { minutos; La Información; } \\
\text { Diario del Alto Aragón. }\end{array}$ \\
\hline
\end{tabular}

Fuente: elaboración propia.

El análisis de los datos, realizado de forma tradicional, comienza mediante codificación abierta, línea a línea, realizándose una primera lectura de aproximación a los textos en la que se señalan palabras y/o frases que destacan por su fuerza descriptiva sobre el fenómeno investigado, verificándose así la presencia de temas o conceptos en un contenido y "su sentido dentro de un texto en un contexto" (Arbeláez y Onrubia, citados en Díaz Herrera, 2018, p. 126).

La categorización se ha llevado a cabo de manera inductiva (las categorías son generadas a partir de los datos, no existiendo categorías iniciales predeterminadas) registrándose tres categorías principales basadas en las cuestiones que la prensa aborda sobre la brecha digital: causas, consecuencias y posibles soluciones. 


\section{RESULTADOS Y DISCUSIÓN}

El total de artículos recogidos en este estudio (374) supone que, en España, durante el lapso de tiempo en el que se despliega este trabajo (primer mes de estado de alarma), se han publicado una media de 12.47 artículos diarios abordando la brecha digital educativa, mostrando el interés de la prensa por la temática.

La prensa de tirada nacional plantea la cuestión desde una perspectiva global, mientras que los rotativos autonómicos y locales concretan los problemas derivados de la brecha digital en sus respectivos territorios de influencia.

\section{Ejemplo 1:}

"Según los datos que maneja el Gobierno [...] el 14\% de los hogares con menores carece de ordenador, un porcentaje que se incrementa hasta el $30 \%$ en los hogares pobres, por lo que apremia a dar cobertura digital al alumnado sin portátil ni conexión" (Los centros escolares cerrados suman 100.000 portátiles sin uso, 10/4/2020, La Nueva España).

\section{Ejemplo 2:}

"[...] solo un $52 \%$ del alumnado ceutí de los colegios públicos tienen medios para desempeñar enseñanzas por internet" (Vivancos, 12/4/2020, El Foro de Ceuta).

En cuanto a las fuentes a las que acude la prensa o de las que se hace portavoz, se encuentra un amplio abanico representativo de la sociedad: instituciones administrativas y de gobierno, asociaciones/federaciones de madres y padres de alumnos, profesorado, sindicatos, partidos políticos, ONGs, fundaciones y asociaciones civiles.

\section{Ejemplo 3:}

"La Confederación de Federaciones de Asociaciones de Padres y Madres de Canarias ha alertado este martes de que la brecha digital impide a muchos niños formarse en casa durante la cuarentena impuesta ante la pandemia de coronavirus Covid-19" (Ampas avisan de que brecha digital impide a muchos alumnos formarse en casa, 7/4/2020, La Vanguardia).

Entre los periódicos consultados destaca La Vanguardia como el medio con mayor número de artículos dedicados a la educación y la brecha digital (figura 1).

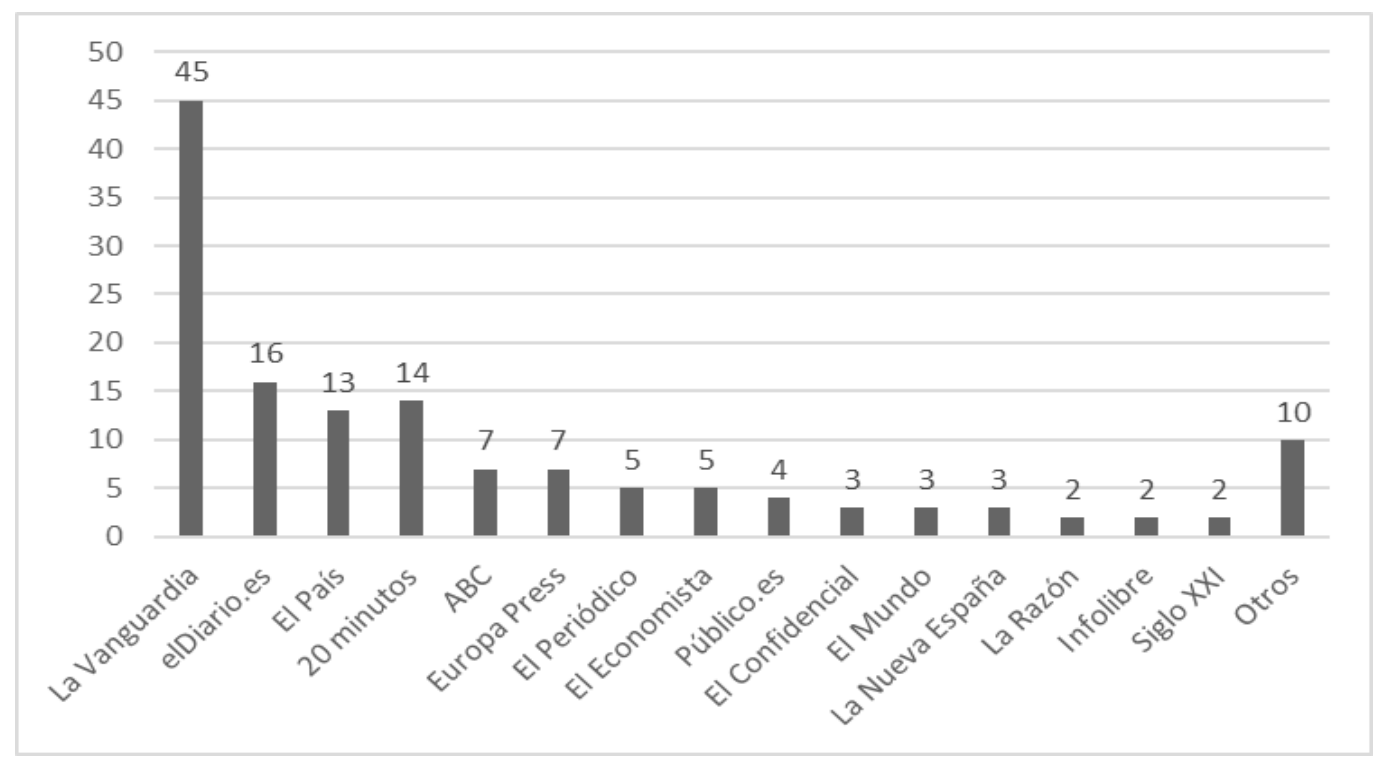

Figura 1. Distribución de textos recogidos en la prensa de tirada nacional. Fuente: elaboración propia 


\subsection{Causas}

Las causas que la prensa refiere como explicativas de la brecha digital están ligadas al contexto del alumnado (ver tabla 2).

Tabla 2. Resumen de las causas indicadas por la prensa

\begin{tabular}{|c|c|c|}
\hline \multicolumn{3}{|c|}{ Contexto del alumnado } \\
\hline \multirow{17}{*}{$\begin{array}{l}\text { Causas explicativas } \\
\text { de la brecha digital }\end{array}$} & \multirow{11}{*}{ Familia } & -Nivel socio-cultural y económico bajos \\
\hline & & -Precariedad laboral + ERTES \\
\hline & & $\begin{array}{l}\text {-Entornos desfavorecidos en exclusión o riesgo } \\
\text { de exclusión social }\end{array}$ \\
\hline & & -Teletrabajo \\
\hline & & -Competencia digital \\
\hline & & -Sin red familiar de apoyo \\
\hline & & -Familias monomarentales \\
\hline & & -Familias migrantes \\
\hline & & -Familias numerosas \\
\hline & & -Familias de etnia gitana \\
\hline & & -Factor tiempo \\
\hline & \multirow{4}{*}{ Recursos } & -Falta o limitación de equipamiento \\
\hline & & -Sin conexión a Internet, sin banda ancha \\
\hline & & -Condiciones materiales del hogar \\
\hline & & $\begin{array}{l}\text {-Disparidad de recursos y digitalización de } \\
\text { centros escolares }\end{array}$ \\
\hline & \multirow[t]{2}{*}{ Entorno } & $\begin{array}{l}\text {-Rural: inexistencia o inestabilidad de red de fibra } \\
\text { óptica. }\end{array}$ \\
\hline & & -Urbano: según barrios. \\
\hline
\end{tabular}

Fuente: Elaboración propia

El hecho de que el alumnado no pueda asistir a los centros educativos, ha implicado que la familia -además de ser el entorno cardinal de desarrollo personal, socialización y cuidados en su sentido más amplio- se convierta en la estructura que soporta, también, la educación (Muñoz y Lluch, 2020).

Los artículos analizados ponen el acento en la situación de niños y niñas de familias desfavorecidas, incidiendo en la precariedad laboral y rentas bajas, a las que ahora se suman los expedientes de regulación temporal de empleo (ERTE), y en las que tienen en la economía sumergida o informal su única fuente de ingresos familiares. Asimismo, se reseñan condiciones y circunstancias vividas en muchos hogares:

1. Escasez o falta de alimentos, paliado en parte por las instituciones y la colaboración de distintas entidades sin ánimo de lucro, asociaciones civiles y voluntariado.

2. Falta de condiciones materiales primarias del hogar: servicios básicos como la luz, el agua, o la calefacción.

3. Espacios físicos no adecuados (ventilación, iluminación o tamaño y distribución de la vivienda) y número de personas que comparten la vivienda. 


\title{
Ejemplo 4:
}

"Lo académico es lo menos importante, se puede recuperar con el paso del tiempo. [...] Lo que más nos preocupa es que este sistema de tele-formación está poniendo encima de la mesa las desigualdades brutales del alumnado" (Rodríguez-Álvarez, 26/3/2020, InfoLibre).

Se replantea la brecha digital en su relación con la pobreza energética y/o con el sobreesfuerzo o capacidad de pago de los servicios esenciales: si apenas puede mantenerse un nivel mínimo de confortabilidad dentro del hogar, la conexión a Internet es un gasto prescindible. La escasez o la carencia de recursos tecnológicos y/o conectividad necesarios para soportar la modalidad educativa online, la disparidad digital de los centros escolares 0 disponer un único ordenador por familia son algunas de las implicaciones de la fragilidad económica de las familias. En estas circunstancias, convertir el hogar en aula y proseguir con expectativas de éxito y naturalidad el proceso de enseñanza-aprendizaje es un desafío complicado.

\begin{abstract}
Ejemplo 5:
"Hay familias que no tienen ordenadores en casa. Otras que tienen que compartirlo entre todas las personas del hogar. $Y$ otras que ni siquiera tienen internet. Un problema que se puede acentuar con la oleada de ERTE y despidos que está provocando la pandemia del coronavirus: ya había familias que no tenían acceso a wifi por sus condiciones económicas y, en un escenario de crisis, la situación empeora. Si no tienes para llegar a fin de mes, no tienes para conectarte a la red. La realidad es cruda, pero muestra una vez más que en la educación tampoco hay igualdad de oportunidades" (Asuar, 18/3/2020, Público).
\end{abstract}

La geografía también contribuye a profundizar la brecha digital (Cabero y Ruíz, 2018; Torres, 2017 o Vázquez, 2017). De especial calado en periódicos de ámbito local, se denuncia la inexistencia o inestabilidad de las redes de fibra óptica y de banda ancha en zonas rurales, despobladas o de difícil orografía, repercutiendo no solo en el alumnado que reside habitualmente en ellas, sino también en aquellos que regresan a sus pueblos tras el cierre de centros educativos.

\section{Ejemplo 6:}

"Normalmente los vecinos tienen que salir a buscar algún punto con cobertura, y ahora lo tienen que hacer, con el móvil y el portátil, para descargar los deberes de los niños, para que puedan estudiar igual que los demás. Es indignante que por vivir en un pueblo no tengan los mismos derechos" (Mantecón, 30/3/2020, El Periódico de Aragón).

En el medio urbano, la brecha puede mapearse por barrios, siendo aquellos con población en riesgo de exclusión social los más proclives a sufrirla.

\footnotetext{
Ejemplo 7:

"Las asociaciones de vecinos [...] del Sector Sur, sí han confirmado que muchas familias de estos barrios están sufriendo el problema y que este se extiende a diferentes zonas de la ciudad. [...] en muchos casos los padres están más preocupados por la falta de alimentos para sus hijos que por la falta de clases" (Cobos, 2/4/2020, Diario de Córdoba).
}

El factor tiempo es uno de los elementos sustanciales para la conciliación entre la vida laboral y familiar. La prensa habla de familias multitarea (Lucas, 2020) en referencia a las múltiples responsabilidades que estas enfrentan. La suspensión de las clases presenciales 
aporta más carga, física y emocional, a dicha conciliación: los menores no asisten al centro escolar, sus profesores/as les atienden desde la virtualidad y el hogar se ha transmutado en aula, factores que incrementan la necesidad de apoyo a los menores que se han incorporado a una modalidad educativa para la que no cuentan con la suficiente autonomía de respuesta.

\section{Ejemplo 8:}

"[...] Esta modalidad formativa se basa en el autoaprendizaje de los estudiantes. Los menores de edad, en este sentido, necesitan la presencia de una persona mayor que les anime, estimule, controle y tutorice durante el tiempo de interacción de ese niño con un material o entorno virtual" (Bolaños, 13/4/2020, La voz de Lanzarote).

La conciliación subyace como una fisura más, con matiz económico-social, señalándose las dificultades para compaginar el teletrabajo y la atención a los menores (como compartir espacios y equipos) o tener que seguir asistiendo al puesto de trabajo con los menores en el domicilio. No exenta de dificultades, es razonable pensar que el teletrabajo es una coyuntura más favorable que la vivida por familias que no pueden acogerse a esta práctica laboral.

\section{Ejemplo 9:}

"Hay padres que están machacados porque no pueden seguir el ritmo del trabajo o teletrabajo a la vez que tantas tareas de los hijos" (Lanceta, 25/3/2020, Diario de Cádiz).

Además, el tiempo de dedicación al menor en su proceso de enseñanza-aprendizaje en lo virtual o del hecho de poder disfrutar de los recursos tecnológicos necesarios, la prensa también habla de las competencias digitales de las familias para llevar a cabo dicho acompañamiento educativo, describiendo así no solo una brecha de acceso sino, también, de uso.

\footnotetext{
Ejemplo 10:

"La brecha digital está ahí. Para las familias con más nivel económico es más fácil apoyar a sus hijos en las tareas, tanto los recursos como por las habilidades digitales" (El parón de las clases imprime más distancia a la brecha digital educativa, 6/4/2020, Diario de León).
}

El paso obligatorio a la educación virtual obra, subsidiariamente, como evaluador de las competencias digitales de la comunidad educativa. Por un lado, seguir una formación online, como ya hemos indicado, requiere de una madurez y autonomía personal de aprendizaje que en etapas educativas básicas el alumnado todavía no ha alcanzado. Además, el empleo que niños/as y adolescentes hacen de la tecnología en su vida diaria está más vinculado al ocio, lo lúdico y la comunicación social que a su uso como herramienta enriquecedora de su aprendizaje. Por otra parte, el apoyo de la red familiar, si ya es fundamental en la educación presencial para el acompañamiento educativo, se torna esencial en estos momentos en el que el hogar se ha convertido en aula, especialmente, una vez más, en las etapas iniciales.

Algunos artículos ponen su atención en las familias monomarentales como aquellas que puedan estar en un mayor riesgo de desconexión escolar al sumarse el factor de la brecha de género laboral. Como indican algunos informes (OECD, 2020; Parlamento Europeo, 2020) las mujeres sufren en mayor medida la precariedad laboral ocupando puestos de trabajo de menor reconocimiento social que ahora se tienen por esenciales (sector limpieza, personal de establecimientos de alimentación o atención y cuidado de mayores, menores o personas dependientes), por lo que deben acudir a sus puestos de trabajo dejando, aún más aislados si cabe, a sus hijos e hijas en casa. En estas circunstancias, además, la falta de red de apoyo 
familiar, bien previa o debida al confinamiento, es una traba más para el bienestar físico y emocional del menor y, en consecuencia, para su proceso de enseñanza-aprendizaje.

\section{Ejemplo 11:}

"[...], madre soltera, se va todas las mañanas a limpiar los portales de su barrio. Lo hace porque no puede perder el único sustento de su familia. [...] se va cada mañana con su teléfono en el bolsillo, la única herramienta con la que cuentan Lucía y Mario para mantener la tensión escolar. La esperan y, cuando llega, comienzan una carrera contra reloj para no perder comba con sus compañeros de clase" (Ferrero y Mateo, 23/3/2020, El País).

La prensa también destaca la desigualdad, pobreza y exclusión que ya viene soportando la infancia gitana y que se ve agravada por la cuarentena obligatoria. Niñas y niños gitanos parten, en el sistema educativo, con desventaja: la pobreza infantil del colectivo asciende al $89 \%$, con un $51 \%$ de pobreza infantil extrema (Fundación Secretariado Gitano, 2018), un escenario que hace urgente la toma de medidas políticas para proteger a la infancia, garantizar su acceso a la educación y paliar la brecha que el confinamiento hace aún más profunda. Fruto de este escenario de pobreza, se señala que el $79 \%$ de los hogares gitanos no cuenta con ordenador ni con conexión a Internet. A través de distintos programas, la Fundación Secretariado Gitano está ofreciendo orientación y apoyo educativo y buscando alternativas para paliar la brecha digital.

\section{Ejemplo 12:}

"Es urgente adoptar medidas que puedan paliar la brecha que esta situación de confinamiento hace aún más grande para la infancia gitana. [...] La desigualdad de partida se agrava y supondrá una desventaja en el proceso educativo de niñas y niños que tendrán menos oportunidades y estarán en inferioridad de condiciones" (Secretariado Gitano avisa de que la brecha educativa y digital de los niños "se acentúa" con la pandemia del coronavirus, 8/4/2020, EuropaPress).

En condiciones similares se encuentran algunas familias migrantes: precariedad laboral, sin red de apoyo familiar, a lo que puede sumarse la dificultad de no manejar plenamente el idioma.

Ejemplo 13:

"Las familias con un nivel sociocultural más bajo o los inmigrantes que aún tienen problemas con nuestro idioma serán las más afectadas" (El parón de las clases imprime más distancia a la brecha digital educativa, 6/4/2020, Diario de León).

Respecto a las familias numerosas, la prensa destaca las dificultades afrontadas para que sus miembros puedan proseguir con su labor educativa.

\section{Ejemplo 14:}

"Vivimos la brecha digital cada día. Solo tenemos un ordenador de mesa para las cinco. Está en la habitación matrimonial y hacemos turnos para usarlo" (García-Rey, 30/3/2020, El Confidencial ).

\subsection{Consecuencias}

Los textos analizados subrayan que la supresión de la escolaridad presencial repercute, especialmente, en el alumnado que ya se encuentra en situaciones socioeconómicas 
desfavorables o vulnerables, comprometiendo los objetivos de la Agenda 2030 (UNESCO, 2016) por el derecho a una educación en igualdad e incluyente para todos.

\section{Ejemplo 15:}

"Se dejará atrás a toda una serie de chicos y chicas a quienes el sistema ya no ha garantizado de entrada las mismas oportunidades que al resto, y que todavía se agravarán más" (Directora de centro segregado: "proporcionar ordenadores no garantiza equidad" 7/4/2020, La Vanguardia).

Además de aspectos relevantes para el bienestar físico, psicológico y emocional del niño, referidos al proceso educativo propiamente dicho, la UNESCO (2020) señala:

Interrupción del aprendizaje: la escuela garantiza los aprendizajes básicos, su clausura supone el menoscabo de oportunidades para el desarrollo y perfeccionamiento de los niños en general y para aquellos de entornos más desfavorecidos en particular al contar con un menor número de posibilidades de acceder a acciones educativas fuera del contexto educativo formal.

\section{Ejemplo 16:}

"Mientras los niños de familias instruidas tienen estímulos culturales, hay otros que pierden muchos aprendizajes porque la escuela es su único lugar de aprendizaje" (Colell, 30/3/2020, El Periódico).

Falta de preparación y/o competencia digital de las familias para la enseñanza a distancia: las familias se ven forzadas a asumir la atención y acompañamiento del menor en su proceso de enseña-aprendizaje desde sus hogares, labor que plantea no pocas dificultades cuando su propia preparación y/o competencia digital es insuficiente cuando no inexistente. De nuevo, son las familias con menos instrucción educativa y recursos quienes sufren más esta situación.

Desigualdad en el acceso a plataformas de aprendizaje digital: la insuficiencia de acceso a las tecnologías y a la conexión a Internet, por inexistente o deficitaria, supone un obstáculo para la formación educativa. Una vez más, será el alumnado de entornos vulnerables quien más acuse las carencias en su proceso personal de enseñanza-aprendizaje.

Tendencia al incremento de las tasas de abandono escolar: el cierre prolongado en el tiempo de las aulas escolares dificulta lograr que niños y jóvenes regresen a la escuela y permanezcan en el sistema educativo tras su reapertura. En este sentido, Giannini y Albrectsen (2020), inciden en la vulnerabilidad añadida por cuestión de género a nivel mundial.

\section{Ejemplo 17:}

"A día de hoy hay estudiantes que han desaparecido, están desescolarizados porque no se han puesto en contacto con sus escuelas e institutos" (López de Pariza y Fernández, 29/3/2020, El Correo).

Estas perspectivas se trasladan al terreno periodístico publicando inconvenientes y desventajas que el alumnado más vulnerable soporta o soportará: reducción del éxito académico; ampliación de la brecha educativa; dilatación la desigualdad social; repercusión en el rendimiento académico; afectación en las habilidades cognitivas; paréntesis lingüístico para alumnado migrante no hispanohablante; incremento del abandono escolar; probabilidad de repetir curso; escalada del absentismo escolar; y padecimiento de secuelas académicas, sociales, físicas y emocionales.

La escuela, como institución social que asume el reto de mitigar la desigualdad social, ofrece en la presencialidad un entorno seguro e inclusivo en el que la procedencia sociocultural o las capacidades individuales conviven en un espacio colaborativo y de cohesión que vela por la equidad, la igualdad y por garantizar el derecho de todos a la educación (Gobierno de España 2018; Rivas, 2016). 


\subsection{Soluciones}

Expuestas las necesidades y repercusiones para el alumnado, los artículos analizados también proponen algunas medidas a corto y largo plazo, tendentes a atenuar dicha brecha.

Tabla 3. Soluciones propuestas

\begin{tabular}{|c|c|c|c|c|}
\hline \multirow{5}{*}{ Soluciones } & \multirow{4}{*}{ Corto plazo } & \multicolumn{3}{|c|}{$\begin{array}{l}\text { - Televisión educativa } \\
\text { - Utilización de software libre }\end{array}$} \\
\hline & & $\begin{array}{l}\text { Suministro de } \\
\text { recursos }\end{array}$ & \multicolumn{2}{|c|}{$\begin{array}{l}\text {-Préstamo/cesión de dispositivos } \\
\text { digitales. } \\
\text { - Facilitar conexión a Internet. } \\
\text { - Liberar redes wifi }\end{array}$} \\
\hline & & $\begin{array}{l}\text { Medidas } \\
\text { paliativas }\end{array}$ & $\begin{array}{l}\text { De } \\
\text { comunicación }\end{array}$ & $\begin{array}{l}\text { - Correo electrónico. } \\
\text { - Aplicaciones de } \\
\text { comunicación. } \\
\text { - Vía telefónica. } \\
\text { - Correo postal: } \\
\text { entrega y recogida de } \\
\text { actividades. }\end{array}$ \\
\hline & & & $\begin{array}{l}\text { De } \\
\text { desempeño }\end{array}$ & $\begin{array}{l}\text { - Tareas no } \\
\text { dependientes de la } \\
\text { tecnología. } \\
\text { - No avanzar } \\
\text { contenidos. } \\
\text { - Abrir centros } \\
\text { escolares en verano. }\end{array}$ \\
\hline & Largo plazo & \multicolumn{3}{|c|}{$\begin{array}{l}\text { - Digitalización de la escuela } \\
\text { - Coalición mundial UNESCO } \\
\text { - Políticas de digitalización e informatización de los hogares } \\
\text { desde el gobierno central. } \\
\text { - Inversión en infraestructuras y redes wifi. } \\
\text { - Actualización continua de sistemas en centros educativos. } \\
\text { - Formación de profesionales en programas }\end{array}$} \\
\hline
\end{tabular}

Fuente: Elaboración propia

A corto plazo, además de las medidas y disposiciones de las instituciones públicas, cabe destacar la colaboración de distintos sectores de la sociedad: empresas del sector de las telecomunicaciones, editoriales, redes vecinales, ONGs, voluntariado, asociaciones 0 fundaciones que están aportando su trabajo, recursos y esfuerzos para cerrar, en la medida de sus posibilidades, la brecha digital de acceso.

Con carácter urgente, algunas de las iniciativas puestas en marcha han sido las siguientes:

Televisión educativa. Implementación a nivel estatal, desde el Ministerio de Educación y Formación Profesional y Radio Televisión Española, del proyecto "Aprendemos en casa» a través de la televisión pública. A nivel autonómico, esta medida también se ha puesto en marcha en comunidades como Cataluña, Castilla y León o Castilla-La Mancha. Desde sectores educativos se advierten críticas en dos vertientes: por un lado, hacia la calidad de los contenidos y las metodologías ofrecidas, y, por otro, la condición necesaria de acompañamiento que guíe y oriente el proceso de enseñanza-aprendizaje, especialmente para el alumnado con necesidades educativas especiales.

Software libre. Se aconseja la utilización de paquetes educativos y aplicaciones de software libre y acceso gratuito por cuanto contribuye a suavizar la brecha digital en contraposición al software de propietario.

Suministro de recursos. Préstamo, cesión o reutilización de ordenadores, móviles y/o tabletas digitales, y suministro de conexión a Internet (routers, bonos wifi, receptores wifi por USB y/o tarjetas SIM). Esta medida se pone en marcha desde las consejerías de educación o ayuntamientos y con la colaboración de empresas, entidades privadas y, distintos colectivos y asociaciones. Como paso previo, se estipula la identificación del alumnado, mediante encuestas o datos aportados por los centros educativos, susceptible de encontrarse en situación de desconexión. La crítica a estas iniciativas apunta a que la distribución de 
ordenadores o proporcionar conexión no suple los problemas de equidad e igualdad preexistentes. Se incide en que este alumnado ya parte de posiciones de desventaja y tendrá consecuencias para su aprendizaje y desarrollo psicosocial. Acudir al centro educativo supone para muchos niños y niñas estar conectados a la rutina escolar y acceder a actividades estimulantes a las que no tienen acceso en su entorno más próximo. Por ello, organizaciones como Save the Children señalan la imperiosa necesidad de apoyo y seguimiento por parte de los centros educativos (Borraz, 2020).

Medidas paliativas. Considerándose que las familias disponen al menos de un Smartphone, se propone el uso de correo electrónico y otras apps sociales para mantener la comunicación con el alumnado, asegurar su vinculación con la escuela y continuar con su formación académica. La reprobación a esta medida alude a la calidad de la experiencia digital a través de teléfono móvil. La utilización del correo postal y la colaboración de voluntarios y redes vecinales actúan como mediadores entre el centro educativo y el alumnado para la distribución y recogida de material escolar y tareas offline.

Ejemplo 18:

"Cantabria ha estrenado una nueva aplicación, bajo el nombre de 'DonoMiWifi', que permite compartir la conexión a internet entre vecinos y que nace con el objetivo de combatir el aislamiento y evitar que ningún estudiante se quede sin acceso a la educación online durante la cuarentena" (Estrenan una app para compartir Wifi entre vecinos en Cantabria, 6/4/2020 El Confidencial).

Desempeño. Se recomienda establecer tareas escolares competenciales y motivadoras no dependientes de la tecnología, acortar el currículo o no avanzar en contenidos. Una propuesta es la apertura de escuelas durante el verano para ofrecer actividades de refuerzo, lúdicas y culturales.

\section{Ejemplo 19:}

"No puede basarse exclusivamente en recursos digitales que exigen un ordenador, conexión a internet y una impresora, además de dar por hecho que todas las familias tienen el capital cultural y el tiempo físico para enseñar a cada menor" (EuropaPress, 13/4/2020).

A largo plazo comienzan a proyectarse algunas iniciativas que han de activarse para reducir la brecha digital. Por ejemplo, la digitalización de la escuela, la adhesión a la coalición de la UNESCO, la implementación de políticas de digitalización e informatización de los hogares, la inversión y creación de infraestructuras y redes adecuadas, la actualización continua de sistemas en los centros educativos, la formación de profesionales en programas, la formación permanente del profesorado y la extensión de la formación en competencia digital a las familias.

Igualmente, vertebrar políticas sociales y educativas que aborden la inversión y la bajada de ratios, entre otras, para contrarrestar el desfase académico al que pueda verse expuesto el alumnado más vulnerable, asumiendo, además, que la educación en modalidad online puede regresar en próximos cursos académicos.

\section{CONCLUSIÓN Y REFLEXIÓN}

Las diferencias sociales entre el alumnado y la elevación de las situaciones de vulnerabilidad como uno de los factores de riesgo más acuciantes para el abandono y el fracaso escolar (Navarrete, 2007; Prieto, 2015) eran ya una realidad bien conocida por quien está a pie de aula. Para la opinión pública, en cambio, las graves dificultades, cuando no imposibilidades, que el alumnado debe afrontar para desarrollar su formación académica cobran más protagonismo, al ponerse explícitamente de manifiesto por las especiales circunstancias que se viven y acaparando más portadas, artículos de información u opinión, entrevistas y/o editoriales. 
En lo educativo, la desigualdad digital supone seguir cavando el foso de la exclusión, una exclusión que ahora se hace también digital; en una sociedad eminentemente tecnológica, quedarse fuera supone una traba más para desempeñarse como ciudadanos del siglo XXI. Hoy se resiente su vida escolar, mañana se resentirá su vida laboral, social, política, participativa y cultural, cerrándose un círculo que indica que a menor preparación académica mayor probabilidad de fragilidad socio-económica y exclusión.

Este estudio confirma la importancia de la función de la escuela como institución frente a las desigualdades; una función que se ha visto socavada por la falta de presencialidad y que afecta a aquellos más vulnerables y con menor acceso a lo digital. La brecha digital es un problema de raíz profunda que actúa como indicador colateral de la desigualdad social y de las distintas condiciones de partida y de oportunidades desde las que el alumnado emprende su trayectoria escolar (Cabero y Ruiz, 2018; Olarte, 2017).

La prensa, potente agente socializador, marca los temas de debate público, ejerciendo influencia en las agendas sociales y políticas. Aun con sus limitaciones e interpretaciones parciales o subjetivas, la prensa ha sido capaz de exponer a la opinión pública un problema socio-educativo que va más allá de la discusión simplificada sobre tecnología educativa, expone la nueva desigualdad social generada por lo digital. Ha sido capaz de crear debate político y controversia social e imaginar soluciones para la construcción de una realidad digital inclusiva, dando así por buena su contribución a visibilizar y sensibilizar sobre esta causa.

Finalizamos este estudio señalando algunas de sus limitaciones. En primer lugar, realizar una revisión de temática socio-educativa a través de la prensa puede implicar que el objeto de estudio se vea abordado desde una perspectiva incompleta. Además, no se han revisado publicaciones especializadas en el ámbito de la educación. Corresponde señalar, asimismo, que los datos han sido recogidos durante el primer mes de confinamiento, aunque la prensa ha seguido publicando conforme a las nuevas circunstancias y decisiones tomadas por las administraciones (por ejemplo, las relativas a evaluación).

Como futuras líneas de investigación se propone: a) realizar estudio para comparar, confrontar y cotejar estos resultados con datos obtenidos a través de estudios publicados en revistas científicas; b) extender la obtención de datos en temporalidad, analizando documentos que abarquen todo el periodo en el que se ha mantenido la educación online y c) comparar con países del entorno cómo se ha abordado por parte de la prensa esta situación.

\section{ENLACES}

Enlace a escritorio Symbaloo: https://bit.ly/2yF2cbH utilizado como registro de datos.

\section{REFERENCIAS BIBLIOGRÁFICAS}

Albert, M.J. (2009). La investigación educativa: claves teóricas. McGraw Hill.

Alva, A.R. (2015). Los nuevos rostros de la desigualdad en el siglo XXI: la brecha digital. Revista Mexicana de Ciencias Políticas y Sociales, 60, 265-286. http://dx.doi.org/10.1016/S0185-1918(15)72138-0

Ampas avisan de que brecha digital impide a muchos alumnos formarse en casa, (2020, abril 7). La Vanguardia. http://bit.ly/2MenQK7

Andrés, G.D. (2014). Una aproximación conceptual a la "apropiación social" de TIC. Questión $1(43), 17-31$.

Asuar, B. (2020, marzo 18). Las desigualdades sociales se acentúan en la cuarentena educativa. Público.

Bolaños, M.C. (2020, abril 12). Prioridades en la educación en tiempos de emergencia: Máquinas versus personas. Gomera Noticias. https://bit.ly/2X7QQol

Borraz, M. (202, marzo 31). Confinados con la nevera vacía o viviendo en una habitación: la factura emocional de la crisis depende de la clase social. Eldiario.es. https://bit.ly/2WwiiNP 
Burbules, N. (2014). El aprendizaje ubicuo: Nuevos contextos, nuevos procesos. Entramados, 1(1), 131-135.

Burri, M. (2011). Re-conceptualizing the Global Digital Divide. Journal of Intellectual Property, Information Technology and E-Commerce Law, 2(3) 217-224.

Cabero, J. y Palacios, A. (2019). Marco Europeo de Competencia Digital Docente "DigCompEdu". Traducción y adaptación del cuestionario "DigCompEdu Check-In". Edmetic, Revista de educación mediática y TIC, 9(1), 213-234, https//doi.org/10.21071/edmetic.v9i1.12462

Cabero, J. y Ruiz, J. (2018). Las Tecnologías de la Información y Comunicación para la inclusión: reformulando la brecha digital. IJERI: International Journal of Educational Research and Innovation, 9, 16-30.

Calderón, D. (2017). Una aproximación a la evolución de la brecha digital entre la población joven en España (2006-2015). Revista Española De Sociología, 28(1), https://doi.org/10.22325/fes/res.2018.16

Califano, B. (2015). Los medios de comunicación, las noticias y su influencia sobre el sistema político. Revista Mexicana de Opinión Pública, 19, 61-78.

Carneiro, R., Toscano, J.C. y Díaz, T. (Coords.) (2011). Los desafíos de las TIC para el cambio educativo. Santillana.

Cobos, P. (2020, abril 2). Coronavirus en Córdoba: La brecha digital deja atrás a numerosos alumnos. Diario de Córdoba.https://bit.ly/2Wx9jfi

Colell, E. (2020, marzo 30). Las clases 'on line' por el coronavirus lastran a los más pobres. El Periódico. https://bit.ly/3cO1LL4

Díaz-Herrera, C. (2018). Investigación cualitativa y análisis de contenido temático. Orientación intelectual de revista Universum. Revista General De Información y Documentación, 28(1), 119-142. https://doi.org/10.5209/RGID.60813

Díez, E. y Díaz, J.M. (2018). Ecologías de aprendizaje ubicuo para la ciberciudadanía crítica. Comunicar, 54, 49-58. https://doi.org/10.3916/C54-2018-05

Apdha alerta de menores pasando hambre en Andalucía por la crisis y exige que este periodo "no afecte a la nota media. (2020, abril, 13). EuropaPress. https://bit.ly/3dlBEVC

Delgado, J.M y Martínez, L.C. (2017). Envejecimiento y desequilibrios poblacionales en las regiones españolas con desafíos demográficos. Ería, revista de geografía, 37 (1), 21-43

Directora de centro segregado: "proporcionar ordenadores no garantiza equidad". (2020, abril, 7). La Vanguardia. https://bit.ly/3f5ZzA0

Estrenan una app para compartir Wifi entre vecinos en Cantabria. (2020, abril 6). El Confidencial. https://bit.ly/3g2YB87

El parón de las clases imprime más distancia a la brecha digital educativa. (2020, abril 6). Diario de León. https://bit.ly/2VQ9aCw

Fernández, M. (2017). Desigualdades educativas en la sociedad digital. Zoom social. Laboratorio de alternativas. Fundación Alternativas. https://bit.ly/3b5IG53.

Ferrero, B. y Mateo, J.J. (2020, marzo 23). Martina en línea, Lucía desconectada: la brecha escolar que marca el coronavirus. El País. https://bit.ly/2VMQDYG

Fundación Secretariado Gitano (2018). Estudio comparado sobre la situación de la población gitana en España en relación al empleo y la pobreza. Fundación Secretariado Gitano. http://bit.ly/2LcdmKG

García-Rey, M. (2020, marzo 30). El telecolegio acentúa la brecha económica: "Tengo un ordenador para cuatro hijas". El Confidencial. https://bit.ly/3feSQE2 
Giannini, S. y Albrectsen A.B. (2020). El cierre de escuelas debido a la Covid-19 en todo el mundo afectará más a las niñas. Organización de las Naciones Unidas para la Educación, la Ciencia y la Cultura. https://bit.ly/2KM2Nuz

Gobierno de España (2018). Plan de acción para la implementación de la Agenda 2030. https://bit.ly/382aMSN

Gómez-Navarro, D., Alvarado, R., Martínez, M. y Díaz de León, C. (2018). La brecha digital: una revisión conceptual y aportaciones metodológicas para su estudio en México. Entreciencias: Diálogos en la Sociedad del Conocimiento, 6(16). https://doi.org/10.22201/enesl.20078064e.2018.16.62611

Gutiérrez, A. y Tyner, K. (2011). Educación para los medios, alfabetización mediática y competencia digital. Comunicar, 38, 31-39. http://dx.doi.org/10.3916/C38-2012-02-03

Instituto Canario de Estadística (2018). Encuesta sobre características y equipamiento de las viviendas. ISTAC. https://bit.ly/2yUfcKb

Instituto Nacional de Estadística (2019). Encuesta sobre Equipamiento y Uso de Tecnologías de Información y Comunicación en los hogares 2019. INE

Keely, B. (Ed.) (2017). Estado mundial de la Infancia 2017. Niños en un mundo digital. UNICEF https://uni.ct/2SkRxcQ

Lanceta, A. (2020, marzo 25). El difícil equilibrio de la educación en el hogar. Diario de Cádiz. https://bit.ly/2ychm85

López de Pariza, S. y Fernández, M. (2020, marzo 29). La enseñanza online agranda la brecha académica entre alumnos. El Correo. https://bit.ly/2TkqEWU

Los centros escolares cerrados suman 100.000 portátiles sin uso (2020, abril 10). La Nueva España. http://bit.ly/2MemTl1

Lucas, B. (2020, marzo 17). Plataformas que fallan, familias multitarea y brecha tecnológica: el drama de la cuarentena educativa. El País. https://bit.ly/2WHhmX5

Mantecón, F. (2020, marzo 30). Aislados por partida doble. El Periódico de Aragón. https://bit.ly/2YkXXwq

Muñoz, J.L. y Lluch, L. (2020). Consecuencias del cierre de las escuelas por el Covid-19 en las desigualdades educativas. Revista Internaiconal para la Jusitica Social, 9 (3e), 1-17.

Navarrete, L. (2007) (Dir.). Jóvenes y fracaso escolar en España. INJUVE.

OECD (Ed.) (2020). Putting finance to work for gender equality and women's empowerment: The way forward. OECD Development Policy Papers, 25. OECD Publishing, https://doi.org/10.1787/f0fa4d91-en

Olarte, S. (2017). Brecha digital, pobreza y exclusión social. Temas laborales: Revista andaluza de trabajo y bienestar social, 138, 285-313.

Parlamento Europeo (2020). Resolución del Parlamento Europeo, de 30 de enero de 2020, sobre la brecha salarial de género (2019/2870(RSP)). Parlamento Europeo. Accesible en: https://bit.ly/2YYBDJd

Pazo, A.J. y Moragón, M.P. (2018). El despoblamiento en Galicia: la visualización de la "catástrofe". Ager: Estudios sobre la despoblación y desarrollo de áreas rurales, (24). https://doir.org10.4422/ager.2018.02

Pérez, G. (2011). Investigación cualitativa. Retos e interrogantes. La Muralla.

Prieto, B. (2015). El camino desde la vulnerabilidad escolar hacia el desenganche educativo. El papel de las escuelas de segunda oportunidad en la estrategia contra el abandono educativo. Profesorado, Revista de currículum y formación de profesorado, 19 (3), 110-125. https://bit.ly/2YPb6jM 
Rivas, J.I. (2016). La escuela pública ante la desigualdad educativa. Una perspectiva política de la justicia social en el sistema educativo. Conferencia presentada en el I Congreso online internacional sobre desigualdad social y educativa en el siglo XXI. https://bit.ly/2WUgg9N

Rivoir, A. y Escuder, S. (2018). Dispositivos digitales en el hogar: incidencia de las desigualdades y las políticas públicas de acceso a partir de un análisis multivariado. $\begin{array}{lllll}\text { Observatorio } & \left(O B S^{*}\right) & \text { Journal, } & 12 & \text { (3), }\end{array}$ https://doi.org/10.15847/obsOBS12320181109

Rodríguez-Álvarez, S. (2020, marzo 26). El aumento de la desigualdad, la gran amenaza del coronavirus en la escuela online. InfoLibre. https://bit.ly/3aP4Etb

Secretariado Gitano avisa de que la brecha educativa y digital de los niños "se acentúa" con la pandemia del coronavirus (2020, abril 8). EuropaPress. https://bit.ly/3aUa5sB

Tejada, M. y Domínguez, G. (2018). Impacto de las brechas digitales en la población extranjera. Ministerio de Sanidad, Servicios sociales e Igualdad. https://bit.ly/3g69K81

Torres, C. (2017). Sociedad de la información y brecha digital en España. Panorama Social,(25), 17-33. https://bit.ly/30uEe05

UNESCO (Ed.) (2016). Educación 2030: Declaración de Incheon y marco de acción para la realización del objetivo de desarrollo sostenible 4 . https://bit.ly/3g2SQYd

UNESCO (Ed.) (2020). Consecuencias negativas del cierre de las escuelas. https://bit.ly/2AopByq

Vázquez, E. (2018). Brecha digital en Andalucía: TIC, sociedad y territorio. Análisis y propuestas en el ámbito de las infraestructuras [Tesis doctoral, Universidad de Sevilla]. idUS. https://bit.ly/3CIFvEd

Vivancos, S. (2020, abril 12). Solo el 52\% del alumnado ceutí tiene acceso a la enseñanza online. El Foro de Ceuta. http://bit.ly/3b0fQGT

\section{INFORMACIÓN SOBRE LAS AUTORAS}

\section{Belén Suárez Lantarón \\ Universidad de Extremadura}

Profesora del área de DOE. Departamento de Ciencias de la Educación de la Facultad de Educación de la Universidad de Extremadura. Dra. en Psicología y Ciencias de la Educación. Sus líneas e intereses de investigación se enfocan en la tutoría y orientación, la atención a la diversidad y la educación inclusiva y la innovación y tecnología educativa. Ha participado en varios congresos nacionales e internacionales y cuenta con publicaciones en revistas científicas indexadas. Es revisora en varias revistas nacionales e internacionales y miembro de redes nacionales como la Red Universitaria de Tecnología Educativa (RUTE), la Red Interuniversitaria de Profesorado de Orientación (RIPO) y la Red Nacional de Aprendizaje y servicio.

Miembro del grupo de Investigación EduTrasnforma-T: https://opendata.unex.es/investiga/investigadores/a807e19722d0296e10da04c8d7f340f8

Y del Instituto Universitario de Investigación y Prospección Educativa de la Universidad de Extremadura (INPEX) https://opendata.unex.es/investiga/institutos-de-investigacion/INPEX

Research Gate: https://www.researchgate.net/profile/Belen-Suarez-Lantaron

Google Académico:https://scholar.google.es/citations?user=rn7tRAIAAAAJ\&hl=es\&authuser=1

Twitter: @Besulan1

\section{Nuria García-Perales}

Universidad de Extremadura

Profesora en la Facultad de Educación (UEx) en el área de Teoría e HistoriaDoctora en Innovación en Formación del Profesorado. Su línea de investigación se vincula con los nuevos entornos de aprendizaje y la Historia política y social de la educación.

Los textos publicados en esta revista están sujetos a una licencia de Reconocimiento 4.0 España de Creative Commons. Puede copiarlos, distribuirlos, comunicarlos públicamente y hacer obras derivadas siempre que reconozca los créditos de las obras (autoría, nombre de la revista, institución editora) de la manera especificada por los autores o por la revista. La licencia completa se puede consultar en:Licencia Creative Commons Atribución-NoComercial-Compartir por iqual 4.0 Internacional. 